\title{
Les variations de la température estivale dans une paroi rocheuse du Jura
}

\section{Conditions de l'experience:}

Ces mesures ont été effectuées pendant le mois de juillet 1973 dans une carrière abandonnée du Jura neuchâtelois. L'endroit choisi est situé près de la Vue des Alpes (coord. 557,07/ 213,78 C. N.S. $1: 25^{\prime} 000$ ) à $1280 \mathrm{~m}$ d'altitude dans une paroi rocheuse verticale orientée au sud. Le calcaire du Kimeridgien y est massif, peu diaclasé, parcouru de joints de stratifications espacés. (Certaines strates ont plus d'un mètre d'épaisseur). La roche est grisâtre, ternie par la patine et la colonisation de lichens.

Les variations de la température ont été enregistrées à l'aide de thermosondes à platine montées sur verre et collées dans des tubes d'acier inoxydable (de $8 \mathrm{~mm}$ de diamètre et de $4 \mathrm{~cm}$ de longeur). Ces tubes d'acier offrent une protection efficace aux sondes. Au fond des trous $(8,3 \mathrm{~mm}$ de diamètre) préalablement percés on a introduit un mélange de graisse au silicone et de poudre d'aluminium afin d'établir un bon contact thermique entre le milieu rocheux et les sondes. Une fois mises en place les sondes sont isolées de l'extérieur par un colmatage de graisse au silicone. La disposition des sondes (en surface, puis successivement à $6,12,25,50$ et $100 \mathrm{~cm}$ ) permet de suivre l'évolution de la température à la surface, dans les zones proches de celle-ci, comme dans les couches plus profondes de la paroi.

Pour mesurer la température de la surface rocheuse nous avons aménagé dans la paroi une petite saignée, afin d'y loger la thermosonde. Le contact thermique fut réalisé là aussi à l'aide du mélange de poudre d'aluminium et de graisse au silicone. Afin d'éviter l'effet de l'insolation directe sur le tube d'acier. nous l'avons "protégé" par une petite plaquette de liège (dimensions $40 \times 10 \times 2 \mathrm{~mm}$ ) collée contre la partie extérieure du tube, la face extérieure de la plaquette de liège étant elle-même recouverte d'un feuillet d'aluminium réfléchissant la lumière.

La température de l'air a été mesurée dans un abri météorologique. En outre un héliographe complétait l'installation, fournissant d'utiles renseignements.

Un potentiomètre à voies multiples permettait l'enregistrement continu et automatique des températures. (1) La précision des mesures est de $+/-0,30 \mathrm{C}$.
Ces mesures ont pour but d'étudier les variations thermiques dans une paroi rocheuse, en perspective d'une étude de l'incidence morphologique des oscillations thermiques. Dans le Jura, peut-on attribuer à l'action de la thermoclastie certaines fissurations ou desquamations de la roche comme c'est le cas dans les régions chaudes et arides? Question difficile à laquelle nous n'avons pas la prétention d'apporter une réponse. (2)

Les deux extraits de nos enregistrements (fig. 1 et 2) sont intéressants du fait qu'ils sont des échantillons types du climat jurassien estival.(3)

Un jour ensoleillé entrecoupé d'une averse

Le 5 juillet 1973 les conditions météorologiques étaient les suivantes:

température: journée chaude $\left(25^{\circ} \mathrm{C}\right.$. à $13 \mathrm{~h}$.

à La Chaux-de-Fonds).

ciel: $\quad$ clair et ensoleillé jusqu'à $15 \mathrm{~h}$; ensuite très nuageux à couvert.

précipitations: dès $15 \mathrm{~h}$. $45 \mathrm{mn}$., pendant $1 \mathrm{~h}$., orage avec averse mêlée de grêle. (11, $4 \mathrm{~mm}$ de précipitations). vent: $\quad$ fort pendant l'orage, faible le reste de la journée.

Quels ont été les changements de température de la roche?

Une observation attentive des courbes (fig. 1) nous montre que:

- Dès le lever du soleil la surface rocheuse s'échauffe brusquement. Entre celle-ci et $6 \mathrm{~cm}$ de profondeur le gradient atteint $2{ }^{\mathrm{O}} \mathrm{C}$. par $\mathrm{cm}$ à $14 \mathrm{~h}$; puis, au moment de l'averse, il s'annule brutalement. De ce fait, la zone superficielle est soumise à des contraintes variables relativement importantes.

- La brusque inversion thermique due à l'averse occasionne d'autres contraintes tout aussi importantes.

- L'amplitude thermique journalière décroît rapidement avec la profondeur. Elle est de $23^{\circ} \mathrm{C}$. en surface, $11^{\circ} \mathrm{C}$. à $6 \mathrm{~cm}, 10^{\circ} \mathrm{à}$ $12 \mathrm{~cm}, 4,5^{\circ}$ à $25 \mathrm{~cm}, 2^{\circ}$ à $50 \mathrm{~cm}$ et $1,5^{\circ}$ à $100 \mathrm{~cm}$. L'amplitude de la température de l'air est de $9,50 \mathrm{C}$.

- Parallèlement à la décroissance de l'amplitude on note également un certain "retard" dans la transmission de la chaleur. (4) Ainsi,

Prof. Andrej Pancza, 2056 Dombresson 
en surface, la température maximum est atteinte à $14 \mathrm{~h}$. (à $6 \mathrm{~cm}$ également); à $12 \mathrm{~cm}$ à $15 \mathrm{~h} .20 \mathrm{mn}$; à 25 et à $50 \mathrm{~cm}$ aux environs de $17 \mathrm{~h}$; à $1 \mathrm{~m}$ de profondeur à $16 \mathrm{~h}$. $30 \mathrm{mn}$ ! (anomalie due à une diaclase située à $82 \mathrm{~cm}$ de profondeur).

Un changement de temps

Conditions météorologiques du 16 juillet: température: journée assez chaude $\left(19^{\circ} \mathrm{C}\right.$. à $13 \mathrm{~h}$. à La Chaux-de-Fonds). ciel: légèrement voilé se couvrant progressivement en fin de journée.

précipitations: quelques gouttes de pluie par intermittence dès $19 \mathrm{~h}$. Pluie plus intense pendant la nuit (entre $1 \mathrm{~h}$. et $6 \mathrm{~h}$. du matin: $20 \mathrm{~mm}$ ).

vent: variable et modéré.

17 juillet: ciel couvert, vent variable et modéré, apparition du soleil (20 mn.) au milieu de l'après-midi.

La comparaison des températures enregistrées le 16 juillet (fig. 2) avec celles du 5 juillet (fig. 1) est significative et nous suggère les remarques suivantes:

- Le réchauffement plus modéré de la surface rocheuse (fig. 2) s'explique par: le ciel légèrement voilé, la température de l'air plus basse et aussi par un vent plus fort.

- Lors d'un réchauffement moins brusque le gradient reste aussi plus faible $\left(1^{\circ} \mathrm{C}\right.$. par $\mathrm{cm}$ dans la couche supérieure). Par conséquent les contraintes se trouvent fortement atténuées.

- Un jour couvert (17 juillet) l'amplitude thermique de la surface rocheuse est de même importance que celle de l'air. Là, les contraintes sont très faibles ou nulles.

Nos enregistrements de températures publiés ici ne représentent pas des valeurs extrèmes mais plutôt des cas qui se présentent fréquemment dans le Jura, et qui finalement, sont susceptibles d'ébranler la masse rocheuse. Combien de jours enśoleillés faut-il pour fissurer ou faire éclater une roche saine? "Probablement plus de $10^{\prime} 000^{\prime \prime}$ pense J.P. COUTARD qui effectue des recherches de thermoclastie au Centre de Géomorphologie. "Là, dit-il, intervient la notion de fatigue des roches soumises un grand nombre de fois et rapidement. à des efforts variables". (5)
Nous souhaitons que d'autres enregistrements puissent se faire dans des roches non calcaires par exemple et sous des climats différents; ces enregistrements devraient permettre des comparaisons intéressantes.

1. Nous tenons à remercier ici M. le Prof. A. JOURNAUX, directeur du Centre de Géomorphologie du C.N.R.S. de Caen d'avoir facilité nos recherches en mettant à notre disposition l'appareillage permettant d'effectuer ces mesures.

2. Vingt-deux échantillons de calcaire jurassien appartenant à 6 faciès sont soumis depuis presque un an (Centre de Géomorphologie de Caen) à un climat artificiel de type saharien. Voir: "Thermoclastie de quelques calcaires jurassiens" J-P. COUTARD et A. PANCZA Bull. Centre Géom. (sous presse).

3. D'autres mesures ont été faites au cours de l'hiver 1972-73. Les résultats obtenus devront paraître dans notre thèse consacrée à l'étude des formations périglaciaires dans le Jura.

4. Des observations analogues ont été faites dans une étude menée en laboratoire: La propagation du froid dans un bloc de calcaire. Par J-P. BENOIST, J-P. LAUTRIDOU, $\mathrm{J}-\mathrm{Cl}$. OZOUF et A. PANCZA. Bull. Centre Geom. NO 18 (sous presse).

5. Cette notion est empruntée à la mécanique générale des matériaux. Voir: Etude mathématique des phénomènes de thermoclastie. Par H. BERTOUILLE Bull. Centre Géom. No 12 .

\section{Conclusion}

Les enregistrements de températures dans une paroi rocheuse orientée au sud nous donnent des renseignements concernant:

- Les relations de la température de l'air et de la roche,

- L'influence de l'ensoleillement direct,

- L'amplitude thermique journalière à diverses profondeurs,

- L'effet des précipitations sur la température de la roche,

- L'importance du vent, mais ceci d'une manière moins explicite. 
Fig. 1 Variations de la température dans une paroi rocheuse orientée au sud. (5 juillef 1973)
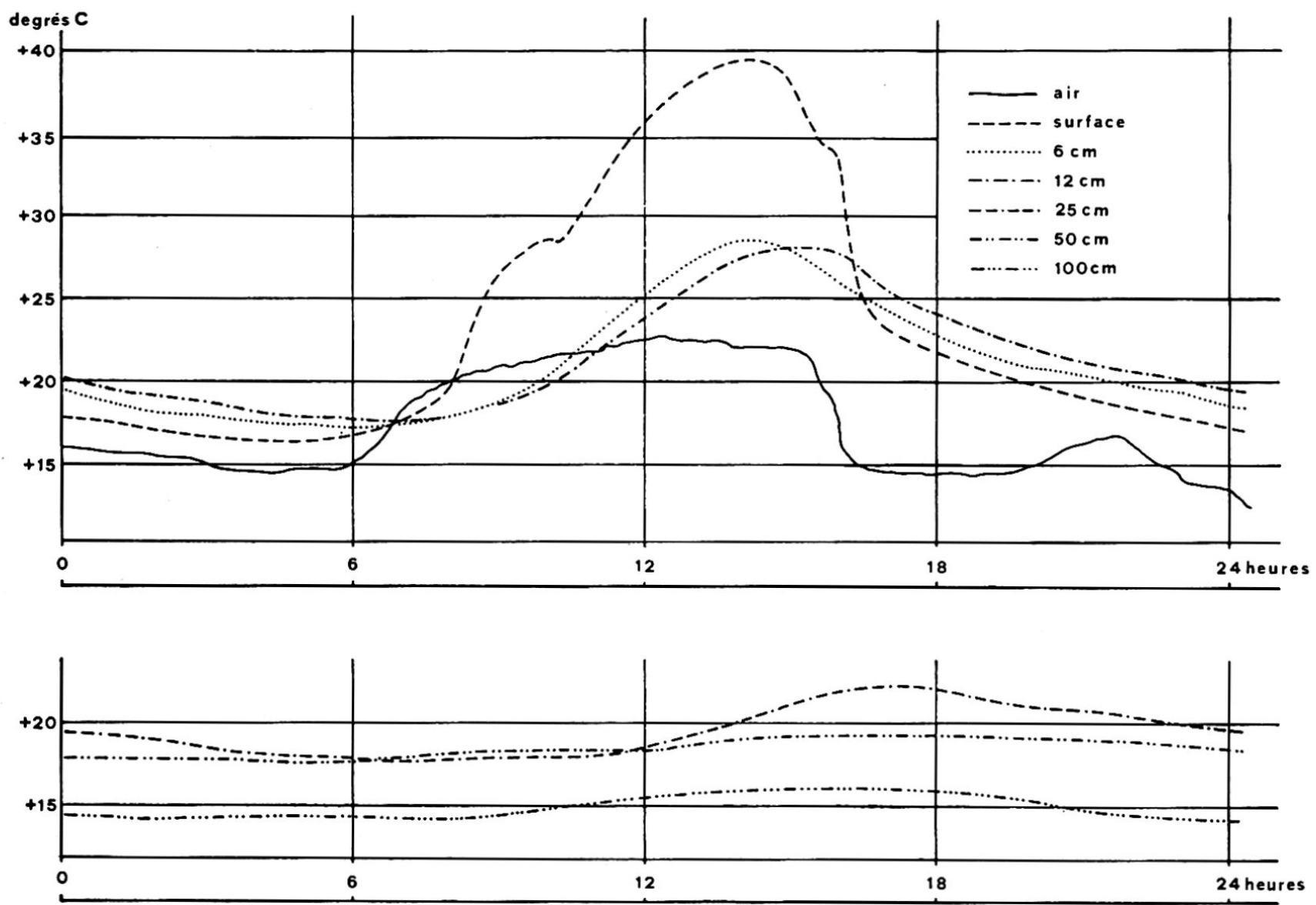

Fig. 2 Variations de la température dans une paroi rocheuse orientée au sud. (16 et 17 juillet 1973)
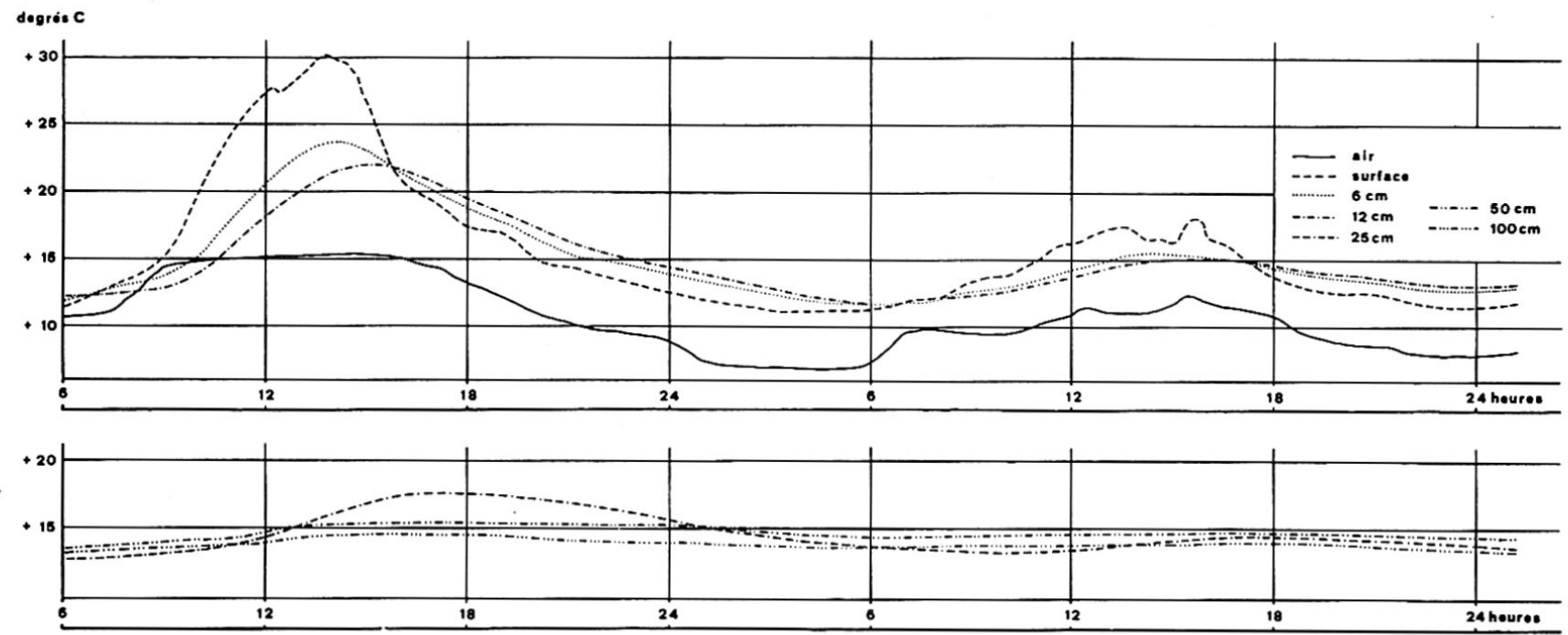


\section{Zusammenfassung}

Im Hohen Jura wurden im Juli 1973 in einer Felswand die Temperaturveränderungen untersucht. Die Messungen erfolgten mit Platinthermosonden und einem Mehrspuren-Potentiometer。

Die hier vorgelegten Ergebnisse stellen keine extremen Fälle dar, sondern spiegeln die sich im Sommer wiederholt einstellenden Verhältnisse wider. Aus den graphischen Uebersichten geht hervor, dass südexponierte Felswände beträchtlichen Temperaturveränderungen unterworfen sind. Ob die Temperaturvariationen aber einen noch nicht angegriffenen Felsen zu schwächen oder gar zu spalten vermögen, lässt sich vorderhand noch nicht eindeutig ermitteln.

\section{Literaturbesprechung}

KUKLINSKI A.: Growth Poles and Growth Centers in Regional Planning, United Nations Research Institute for Social Development. Vol. 5 , 306 S.: Mouton, Paris 1972.

KUKLINSKI A. and R. PETRELLA: Growth Poles and Regional Policies, UN Res. Inst. Soc. Dev., vol. 3: Mouton, Paris, 1972, 267 S.

Der Titel und der Autor, ein sehr bekannter polnischer Geograph, weisen darauf hin, dass diese beiden Publikationen (und weitere des genannten Institutes) für Geographen von grossem Interesse sind. In den beiden vorliegenden Bänden handelt es sich jeweilen um Sammlungen von kürzeren Einzelabhandlungen, die für sich. verstanden sein wollen. $\mathrm{Zu}$ diesem Verständnis ist entweder eine allgemeine Kenntnis der sogenannten growth pole theory notwendig oder ein entsprechender verbindender Kommentar. Letzterer ist freilich ungenügend, was in Verbindung mit der Tatsache, dass fast alle Beiträge unter der heute weitverbreiteten Krankheit leiden, in einer Spezialistensprache geschrieben zu sein, dem an der Sache interessierten Novizen den Zugang ungebührlich erschweren. Growth poles sind die immer zahlreicher und grösser werdenden Städte. L. H. Klaasen schreibt wörtlich: " Die Schaffung von neuen Städten ist ganz genau das, was die Wachstumspolitik (growth policy) anstrebt." Hier wird in wenigen und klaren'Worten gesagt, um was es geht. Ist es wirklich nur - wie Klaasen weiter schreibt - lack of knowledge und political interference, welche uns bisher daran hinderte, die- se Wachstumspole zu den sozio-ökonomischen Optima zu gestalten, die sie nach dieser Theorie sein sollten? Dies scheint uns ein grundlegender Ansatz zu sein, der zum mindesten diskutabel ist. Die andere Schwäche ist offensichtlicher: Es handelt sich um eine Theorie, um ein sorgsam entwickeltes theoretisches Gebäude. Wie jede Theorie kann auch diese ihre Allgemeingültigkeit nur durch Aufgabe von Information erreichen und wird darum, weil das spätere Hinzufügen von Information fast unmöglich ist, nur schwer für die praktische Planung verwendet werden. Wir kommen damit zum Schluss, dass die vorgelegten Arbeiten einen äusserst wichtigen Beitrag zur Weiterentwicklung einer theoretischen Geographie darstellen. Wir machen aber schon Vorbehalte bei der grundsätzlichen Weichenstellung und bezweifeln im Uebrigen den Wert solcher Diskussionen für die praktische Planung, wenn nicht bedeutende $\mathrm{Zu}$ geständnisse gemacht werden.

H. Boesch 